Research Article

\title{
Experimental Evaluation of MR Brake using rollers
}

\author{
Saurabh Kumar*, Dattatraya Zore, Tejas Bhalerao and Vinmay Jagtap \\ †Mechanical Department, Savitribai Phule Pune University, MIT COE Pune India \\ Accepted 02 March 2016, Available online 15 March 2016, Special Issue-4 (March 2016)
}

\begin{abstract}
A generated resistance force in the deformation process is considered to increase the resistance torque of a MagnetoRheological (MR) brake when a variable stiffness material is rolled under the cylindrical form of a roller. This project proposes a new approach to increase the resistance torque of an MR brake using a large-size magnetic particle which can be considered as the roller mentioned above (steel roller or rolling pin). Due to the tapered cylindrical form of the roller and a line contact between the roller and the surface of the motion part, the steel roller can contribute to create a stronger magnetic field and larger resistance force than the conventional one. In this paper, a new MR brake is successfully designed to generate a higher braking torque than the conventional one, which only uses typical MR fluid. To verify the effect of the roller, the proposed MR brake is compared with the conventional one. Both of the MR brakes are designed with the same magnetic circuit and the same material parameters. The performance of the proposed MR brake is compared with that of the conventional MR brake.
\end{abstract}

Keywords: MR brake; MR fluid; Small steel roller; Braking torque; Functional fluid.

\section{Introduction}

A typical MR fluid is a type of functional fluid which has a suspension of magnetic particles in inert carrier liquids. The particles, typically with a size in the order of $1 \mu \mathrm{m}$ to $10 \mu \mathrm{m}$, are added to the fluid, such as mineral or silicone oils. MR fluid also contains small amounts of additives, which affect the polarization of the particles or stabilization of the structure of the suspension to resist settling. However, they may be neglected in modelling the fluid's mechanical response. MR fluid differs from the conventional "magnetic fluid," which contains particles of a much smaller size, typically in the order of $10 \mathrm{~nm}$. The effect of the Brownian motion is greater at this scale, and it prevents the particles from forming fibrils in the presence of the magnetic field.

A new approach to designing an MR brake using a small steel roller was proposed, fabricated to verify the performance of the new and the conventional MR brakes in comparison with the same input conditions. From the experimental results, it was verified that the braking torque of the proposed MR brake was larger than that of the conventional one.

*Corresponding author: Saurabh Kumar

\section{Construction of the proposed brake}

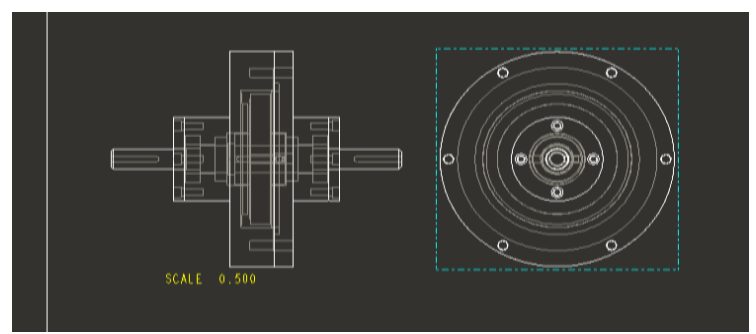

Fig.1 Basic construction of the MR brake using new approach

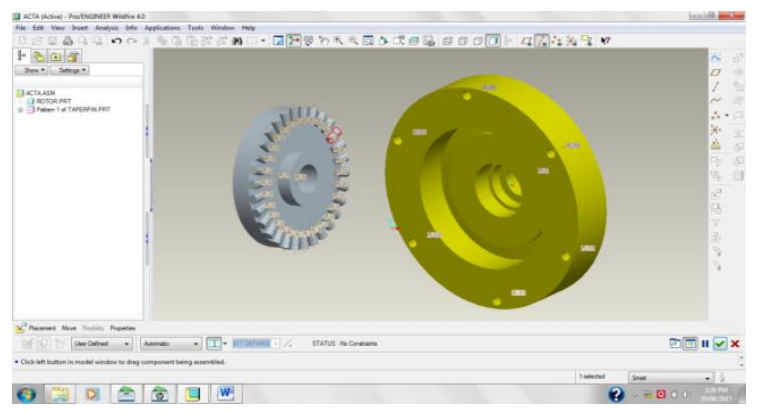

Fig.2 Mounting of the tapered roller on the rotor

\section{Working}

The MR fluid is a smart fluid whose properties can be controlled in the presence of magnetic field. In the absence of magnetic field, the rheological properties of 
the MR fluid are similar to that of base fluid except that it is slightly thicker due to the presence of metal particles.

In the absence of magnetic field, these metal particles align themselves along the direction of flow however when a magnetic field is applied each metal particles becomes a dipole aligning itself along the direction of magnetic field. Thus a chain like structure is formed along the line of magnetic flux which offers mechanical resistance to the flow resulting in an increase in the viscosity of fluid .This mechanical resistance created due to the chain column imparts yield strength to the fluid, making it stiff like a semisolid. This stiffness and hence the yield strength depends on the strength of the magnetic field and also the quality and quantity of metal particles.

MR brake using a small-size steel roller is proposed to achieve the better performance of a semi-active brake based on the theory of hydrodynamic lubrication, plasticity, and electromagnetic. It is desirable to make a higher magnetic permeability to increase magnetic flux and to change the direction of magnetic flux into the MR zones. The MR zone is the space created by the boundary of magnetic pole (wall) and filled with typical MR fluid. These zones have a special form, which is known as the hydrodynamic model and is hardened in the magnetic field. It creates the effect of a resistance force from deformation and a resistance force from hydrodynamic lubrication processes.

When a strong magnetic field is applied, these zones quickly become strong obstacles which resist the motion of the device. Finally, the resistance force of the MR brake clearly increases. Furthermore, this paper proposes the mathematical model for the MR brake based on some of the theories mentioned above.

\section{Modeling of MR Brake}

The characteristics of MR fluid can be described by using a simple Bingham plastic model . The constitutive equation for a Bingham plastic fluid where the total shear stress $(\tau)$ is written as below:

$$
\tau=\tau_{h}+\tau_{p} * \Upsilon
$$

where, $\tau_{\mathrm{h}}$ is the yield stress due to the applied magnetic field, $\mathrm{H}$, and $\Upsilon$ is the shear strain rate. Based on the Eq. (1), the braking torque generated by the friction of the interface between static and moving parts in the MR fluid inside the MR brake can be written as equations;

$$
T_{b}=2 \pi N \int_{r_{i}}^{r_{o}}\left(k H^{\beta}+\mu_{p} \frac{r w_{s}}{h}\right) r^{2} d r
$$

Where $\mathrm{r}$ is the radius of the disk, $\mathrm{s} \mathrm{w}$ is the angular velocity of the rotating disk, $h$ is the thickness of the MR fluid gap between rotor and enclosure, $\mathrm{H}$ is the magnetic field intensity corresponding with $\mathrm{k}$ and $\beta$. The values of $\mathrm{k}$ and $\beta$ are constant by considering the relationship between the magnetic field intensity and the yield stress of the MR fluid.

An integration of Eq. (2) will determine the two types of components of braking torque which are torque generated due to applied magnetic fields $(\mathrm{TH})$ and torque due to friction and viscosity of the fluids( $T$ $\mu$ ). Both torque elements are expressed as follows ;

$$
\begin{gathered}
T_{\mu}=\frac{\pi}{2 h} \mathrm{~N} \mu_{p}\left(r_{o}{ }^{4}-r_{i}{ }^{4}\right) w_{s} \\
T_{H}=\frac{2 \pi}{3} \mathrm{Nk} \alpha\left(r_{o}{ }^{3}-r_{i}{ }^{3}\right) i
\end{gathered}
$$

Therefore, the total braking torque produced by MR brake can be written as follow;

$$
T_{b}=T_{\mu}+T_{H}
$$

\section{Trial and Testing}

Electric motor is connected to the brake and flywheel is mounted on the other side of the brake. Flywheel is carrying weights attached with it to take readings at different load conditions. The supply given to the stator is varied from 1-4 ampere. The conventional MR brake and proposed break is tested for different load to check the efficiency of both the breaks. And finally plots of both brakes after testing are plotted as

(1) Shaft angular velocity response.

(2) Load displacement response.

(3) Torque response.

\section{Tables}

Table 1 Experimental procedure parameters

\begin{tabular}{|l|l|l|l|l|}
\hline \multicolumn{1}{|c|}{ Current (A) } & 1 & 2 & 3 & 4 \\
\hline 5 & $3.6 \mathrm{~s}$ & $1.01 \mathrm{~s}$ & $0.72 \mathrm{~s}$ & $0.6 \mathrm{~s}$ \\
\hline 10 & $>6 \mathrm{~s}$ & $1.52 \mathrm{~s}$ & $1.05 \mathrm{~s}$ & $0.8 \mathrm{~s}$ \\
\hline 15 & $>6 \mathrm{~s}$ & $2.6 \mathrm{~s}$ & $1.31 \mathrm{~s}$ & $1.06 \mathrm{~s}$ \\
\hline
\end{tabular}

\section{Conclusions}

A new approach to designing an MR brake using a small steel roller was proposed, fabricated to verify the performance of the new and the conventional MR brakes in comparison with the same input conditions. From the experimental results, it was verified that the braking torque of the proposed MR brake was 200\% larger than that of the conventional one. A new mathematical model of the proposed MR brake was proposed. As a result of this model, it was found that the operation mode of MR fluid in the new MR brake is different from the conventional shear, valve, or squeeze film mode. In future studies, the different structure of the MR zone should be focused on to improve this study. 


\section{References}

Tran Hai NAM and Kyoung Kwan AHN,(2006) New approach to designing an mr brake using a small steel roller and $\mathrm{mr}$ fluid , Journal of Mechanical Science and Technology 23 , pg no.1911-1923u

Ahmad Zaifazlin. Zainordin, Mohd Azman.(2013) Experimental evaluations on braking responses of magnetorheological brake, International Journal of Mining, Metallurgy \& Mechanical Engineering (IJMMME) Volume 1, Issue 3 .
Professor. Slawomir Bydon, (2002) Construction and operation of magnetorheological rotary brake,Archiwum Process Control Club 2002, poz.20

M. Kciuk ,R. Turczyn, (2006) Properties and application of magneto-rheological fluids,Journal of Achievements in Materials and Manufacturing Engineering, Volume 18 Issue 1-2 September-October 2006. 